\title{
International summit forum in colorectal and anal surgery, Shanghai, China
}

\author{
J. Xu $\cdot$ L. Cui $\cdot$ A. P. Zbar
}

Published online: 24 October 2014

(C) Springer-Verlag Italia Srl 2014

The International Summit Forum in Colorectal and Anal Surgery was recently held in Shanghai at the Xinhua Hospital under the direction of Professor Long Cui on 4-5 July 2014 with specially invited overseas speakers including Professors Francis Seow-Choen (Singapore) and Andrew Zbar (Israel). Professor Seow-Choen spoke about the rise of novel technologies for colorectal surgery in Singapore, focusing on laparoscopic and robotic surgeries and on the new strategy of reverse-TME and hybrid NOTES resections There were also talks on the local Chinese experience with laparoscopic-assisted resection (Drs. Zhen Minhua and Zhongmin, Shanghai), robotic colorectal resection (Dr. Xu Jianming, Shanghai), NOTES (Dr. Fu Tao, Xian) and hand-assisted surgery (Dr. Lin Jianjiang, Hangzhou).

Professor Zbar discussed the technique of transperineal sonography along with novel 3D post-processing endoanal ultrasonographic technology in the delineation of complex fistula-in-ano and for the diagnosis of perianal Crohn's disease. In addition, Dr. Wang Honxiang (Shanghai) outlined the clinical approach to complicated cryptogenic fistula-in-ano. There were further presentations focusing on rectal cancer management (Drs. Liu Menhua, Beijing, and Yao Liqing, Shanghai) with the consideration of the

J. Xu · L. Cui

Colorectal Department of Surgery, XinHua Hospital, Shanghai, China

\section{A. P. Zbar $(\bowtie)$}

Chaim Sheba Medical Center, Tel-Aviv, Israel

e-mail: apzbar1355@yahoo.com

\section{A. P. Zbar}

Department of Anatomy, University of Otago, Dunedin, New Zealand comprehensive care of advanced (T4) rectal cancer (Dr. Fu Chuangang, Shanghai) and excellent presentations of the experience with and outcome of selective inter-sphincteric rectal resection (Dr. Du Jinlin, Jinhua) and extralevator rectal resection (the ELAPE procedure; Dr. Wang Zhenjun, Shenyang).

Additional special presentations included a tactical approach to the diagnosis and management of Crohn's disease (Dr. Gu Yunfei, Liaonin) and a similar evaluation of strategies in the treatment of severe constipation (Dr. Liu Baohua, Beijing). Two further special presentations were made by Professor Long Cui (Shanghai) concerning planning considerations for impending molecular and genetic coloproctological research (given China's considerable resources and facilities for tissue banking) and by Professor Wang Jianping (Guangzhou) who at the start of the meeting outlined the future direction of colorectal surgery in China. The principles highlighted by Professor Wang aim to standardize colorectal resectional techniques across China and to develop a nationwide cooperative colorectal cancer network which will foster clinical research.

The meeting was of high quality and excellently organized with an expanded emphasis on minimally invasive approaches along with the definition of national strategic protocols for the management of inflammatory bowel disease, complex perirectal sepsis and functional bowel disorders. The future of Chinese coloproctology seems assured with a plan spearheaded by Professor Long Cui and his colleagues which will enhance Chinese-led research and will strengthen international collaboration. It is certainly worth placing this congress in one's calendar so as to gain a greater insight into the evolution of Chinese coloproctology and to foster scientific and publishing partnerships. 